\title{
Interactive comment on "Plant functional traits determined the latitudinal variations in soil microbial functions: evidence from a forest transect in China” by Zhiwei Xu et al.
}

Zhiwei Xu et al.

xuzw647@nenu.edu.cn

Received and published: 17 May 2019

The comment was uploaded in the form of a supplement:

https://www.biogeosciences-discuss.net/bg-2018-499/bg-2018-499-AC7-

supplement.pdf 